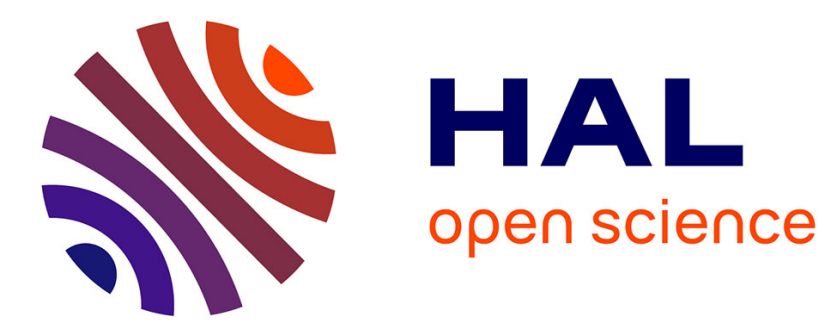

\title{
Sociétés et fluctuations du climat dans les Alpes nord-occidentales au Néolithique moyen \\ Pierre-Jérôme Rey
}

\section{To cite this version:}

Pierre-Jérôme Rey. Sociétés et fluctuations du climat dans les Alpes nord-occidentales au Néolithique moyen. Neige et Glace de Montagne: Reconstitution, dynamique, pratiques, Collection EDYTEM Cahiers de Géographie, nº, pp.37-50, 2009. halsde-00399391

\section{HAL Id: halsde-00399391 https://hal.science/halsde-00399391}

Submitted on 30 Mar 2011

HAL is a multi-disciplinary open access archive for the deposit and dissemination of scientific research documents, whether they are published or not. The documents may come from teaching and research institutions in France or abroad, or from public or private research centers.
L'archive ouverte pluridisciplinaire HAL, est destinée au dépôt et à la diffusion de documents scientifiques de niveau recherche, publiés ou non, émanant des établissements d'enseignement et de recherche français ou étrangers, des laboratoires publics ou privés. 


\section{Collection EDYTEM}

Numéro 8 - Année 2009

Cahiers de

Géographie

\section{NEIGE et GLACE de MONTAGNE}

Reconstitution, dynamique, pratiques

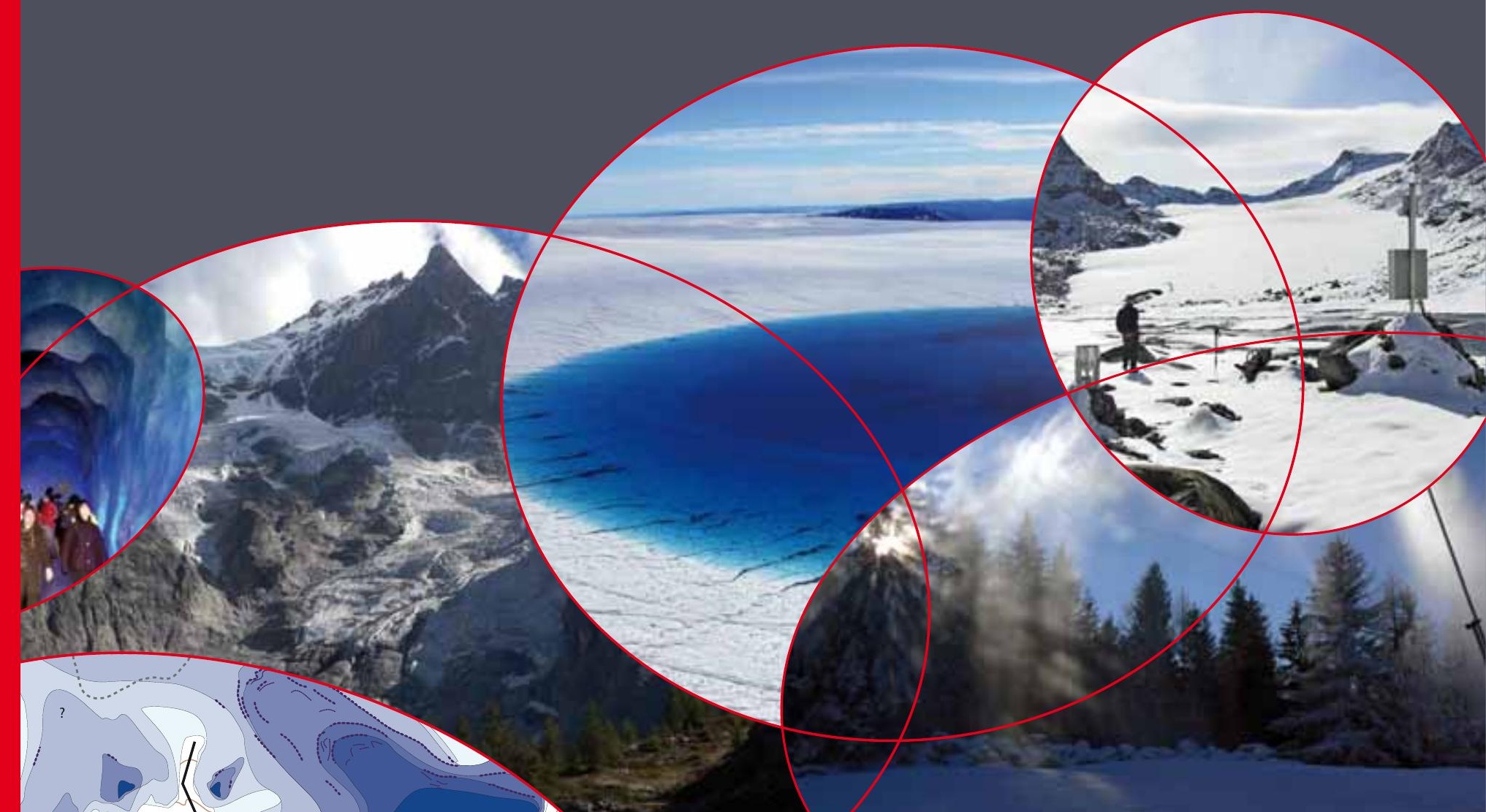





\title{
SOCIÉTÉS ET FLUCTUATIONS DU CLIMAT DANS LES ALPES NORD-OCCIDENTALES AU NÉOLITHIQUE MOYEN
}

\author{
SOCIETIES AND CLIMATE FLUCTUATIONS IN THE NORTH-WESTERN ALPS \\ DURING THE MIDDLE NEOLITHIC
}

PIERRE-JÉRÔME REY

Laboratoire EDYTEM, Université de Savoie/CNRS, Campus scientifique, F 73376 Le Bourget-du-Lac cedex.

Contact : pierjrey@club-internet.fr

\begin{abstract}
RÉSUMÉ
Dans les vallées internes des Alpes nord-occidentales, la densité des sites d'habitats et des nécropoles du Néolithique moyen semble croître durant deux longues périodes d'amélioration climatique. Toutefois, l'état actuel de la documentation ne permet pas de démontrer des fluctuations du peuplement à partir des variations chronologiques du nombre de sites archéologiques. En revanche, les principales phases de rupture ou de changement rapide identifiées dans la culture matérielle, les rites funéraires et l'évolution des réseaux d'échanges montrent des synchronies qui paraissent plus significatives. Elles permettent de proposer l'hypothèse d'un impact croissant des dégradations climatiques sur les évolutions des sociétés humaines, entre le $V^{e}$ et la fin du IV millénaire av. J.-C., en accord avec la probable intensité croissante de ces péjorations.
\end{abstract}

Mots-CLÉS : ALPES, PEUPLEMENT, NÉOLITHIQUE, FLUCTUATIONS CLIMATIQUES, NÉOGLACIAIRE, HABITAT, CULTURES ARCHÉOLOGIQUES, RITES FUNÉRAIRES.

\begin{abstract}
In the inner valleys of the north-western Alps, the density of the middle Neolithic period's settlements and cemeteries seems to increase during two long periods of climatic improvement. However, the present state of our archaeological knowledge does not allow to demonstrate changes in the human occupation, recognized through the chronological variations of the amount of archaeological sites. The main breaks or fast changes identified within the material culture, the funeral practices and the evolution of the exchange networks show simultaneous events which seem more significant. This allows us to suggest the hypothesis of an increasing impact of the climatic degradations on the variations of human societies, between the $5^{\text {th }}$ and the end of the $4^{\text {th }}$ millennium cal BC, following what seems to be the increasing intensity of these degradations.
\end{abstract}

KEYWords: ALPS, OCCUPATION, NEOLITHIC, CLIMATIC CHANGES, NEOGLACIAL, SETTLEMENT, ARCHAEOLOGICAL CULTURES, FUNERAL RITES. 


\section{INTRODUCTION}

L'évolution rapide des référentiels chronologiques et les inquiétudes autour du réchauffement climatique actuel ont favorisé ces dernières années le développement des réflexions sur les liens entre fluctuations du peuplement et dynamiques environnementales (Arbogast et al., 1995 ; Pétrequin et al., 2004 ; Berger 2005). Par l'accentuation des contraintes que procure le relief, les montagnes apparaissent comme un terrain particulièrement favorable pour l'étude de telles interactions.

Dans la vallée alpine de la Tarentaise, une fouille programmée est en cours depuis 1999 sur le site du Chenet des Pierres à Bozel $(950 \mathrm{~m})$, dans un chaos de blocs rocheux implanté sur le flanc nord-ouest du massif de la Vanoise. Les niveaux d'occupations correspondent essentiellement à deux phases du Néolithique moyen, entre 4600 et 4200 av. J.-C. et entre 3900 et 3300 av. J.-C. (Rey 2006) dans une acceptation large des indications livrées par la culture matérielle. Dans la même vallée, un sondage sur le plateau sommital du verrou du Dos de Borgaz à proximité d'Aime a révélé en 2001 une séquence stratigraphique assez comparable (Rey, 2007). La lacune synchrone observée autour de 4100/4000 av. J.-C. dans les niveaux anthropiques de ces deux sites rejoint un constat assez général dans les Alpes françaises. La publication récente de travaux proposant un fort impact des variations climatiques sur les peuplements néolithiques dans des régions d'altitude moindre, comme le Jura (Arbogast et al., 1995 ; Pétrequin et al., 2004) ou la moyenne vallée du Rhône (Berger, 2005), incite à tester la pertinence de cette hypothèse en Tarentaise. La faiblesse des données disponibles impose cependant d'élargir le cadre de réflexion à plusieurs vallées contigües : Beaufortain, Tarentaise, Maurienne, Val d'Aoste, vallée de l'Orco et Val de Suse (figure 1). Ces vallées constituent des

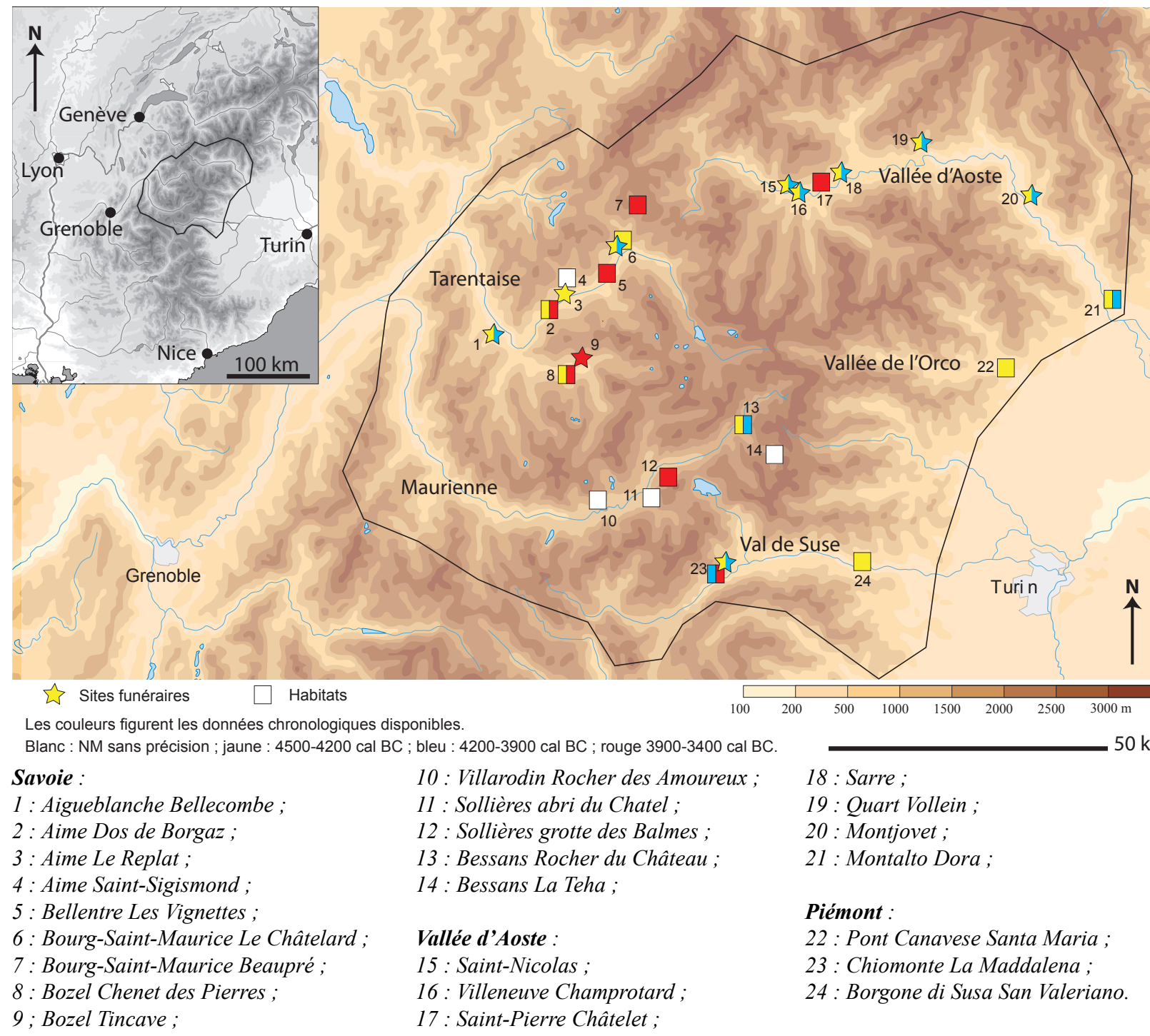

Figure 1 - Localisation de la zone étudiée dans les Alpes nord-occidentales et carte des sites du Néolithique moyen. 


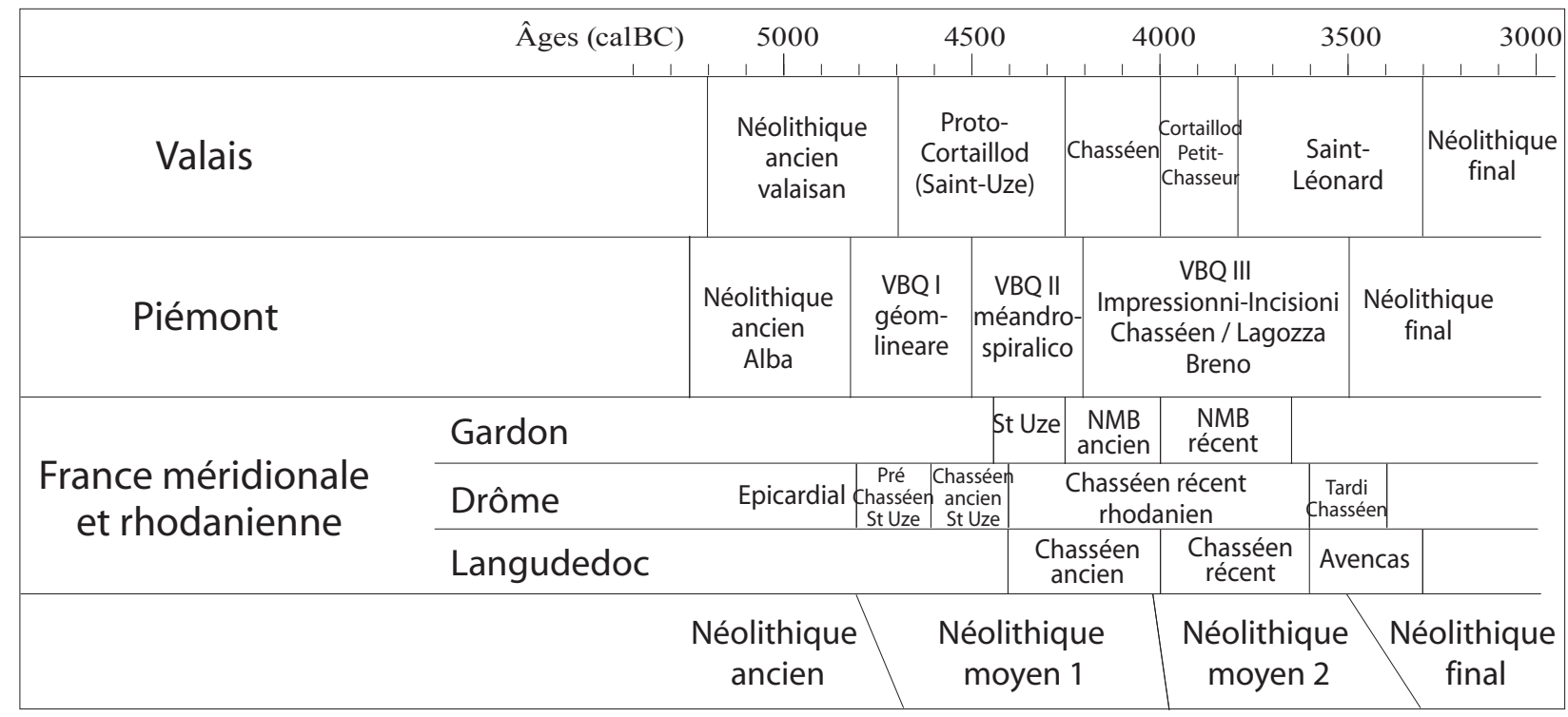

Figure 2 - Cadre chrono-culturel du Néolithique moyen des Alpes nord-occidentales.

axes privilégiés pour les circulations transalpines, dans une portion particulièrement étroite des Alpes internes. La période considérée s'étend de 4800 à 3300 av. J.-C., soit l'acceptation la plus large pour le Néolithique moyen (NM) selon la terminologie et la chronologie en vigueur en Suisse et France du sud. Cette période est classiquement partagée entre Néolithique moyen 1 (NM1) et Néolithique moyen 2 (NM2), la transition intervenant autour de 4000 av. J.-C (figure 2). Les objectifs de ce travail sont de recenser les périodes de changement rapide ou de rupture dans la densité du peuplement, les rites funéraires, la culture matérielle, les pratiques économiques et le fonctionnement des réseaux d'échanges à longue distance. Dans la limite des incertitudes de leur calage chronologique, ces données seront ensuite confrontées aux connaissances sur les fluctuations climatiques holocènes, dont la dynamique des systèmes nivo-glaciaires alpins constitue un marqueur important.

\section{I - OCCUPATION DU TERRITOIRE, PEUPLEMENT ET CULTURES}

Les origines du peuplement néolithique des vallées internes des Alpes nord-occidentales restent floues. Les découvertes du Mésolithique sont rares et se limitent au versant italien. Le Néolithique ancien n'est guère mieux documenté, bien que la diffusion des lames polies en roches vertes d'origine italienne prenne dès cette époque une grande ampleur (Pétrequin et al., 2002 ; Thirault, 2004 a).

Avec un nombre de sites en nette croissance, le Néolithique moyen constitue donc la première phase d'occupation bien documentée dans ces vallées. La documentation disponible, issue de travaux réalisés le plus souvent dans les 40 dernières années, comprend seize habitats et dix nécropoles (figure 1 et tableau 1). Cependant il s'agit très rarement de fouilles de grandes superficies et les résultats souffrent d'un déficit de publication (Rey et Thirault, 1999).

\section{1 - Les sites funéraires}

Les sites funéraires se concentrent principalement en Tarentaise et en Val d'Aoste. La plupart des nécropoles se trouve en fond de vallée, le long du réseau hydrographique principal. Des implantations sur terrasse alluviale sont documentées à Aime et Villeneuve, sur cône torrentiel à Aigueblanche. Les autres sites se trouvent sur des points hauts plus ou moins difficiles d'accès, offrant un bon point de vue sur les axes de communication (Vollein, Chiomonte, Bourg-Saint-Maurice, SaintNicolas).

Comme en Valais (Curdy, 2007), une majorité des nécropoles se situe en dessous de $900 \mathrm{~m}$ d'altitude. Installé à $912 \mathrm{~m}$, Vollein dépasse à peine cette cote. Seuls Saint-Nicolas et Bozel Tincave font exception. Leur implantation entre 1100 et $1200 \mathrm{~m}$ pourrait indiquer un début d'investissement de l'étage montagnard inférieur. Le micro climat local plus chaud et plus sec, ainsi que l'étroitesse du fond des vallées savoyardes et valdôtaines, interviennent vraisemblablement pour expliquer cette différence avec la haute vallée du Rhône.

Quatre des dix nécropoles signalées ont été sondées ou fouillées récemment et fournissent une documentation substantielle bien qu'incomplète : Aime Le Replat (Gély et al., 1991 ; Gély, 2005 ; figures 3 à 5), Quart 


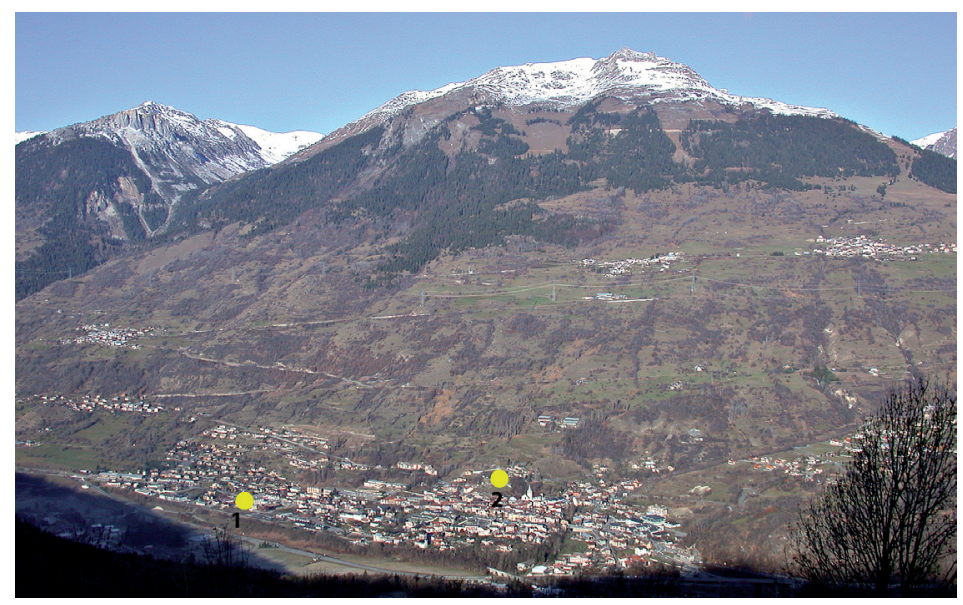

Figure 3 - Vue générale d'Aime en Tarentaise.

1 : nécropole du Replat sur une terrasse alluviale;

2 : habitat sur la colline rocheuse

de Saint-Sigismond.

Dans l'aire «Chamblandes» des groupes régionaux ont été individualisés sur la base des éléments de parure (Moinat et al., 2007). Valais et Val d'Aoste se caractérisent par le dépôt de bracelets en coquilles méditerranéennes, alors que dans le bassin lémanique les inhumés sont accompagnés de pectoraux en défense de suidés, de perles en forme de hache, des boutons à gorge ou de pendeloques rectangulaires en coquille. Les éléments de parures décou-

Vollein (Mezzena, 1997), Chiomonte La Maddalena (Fedele, 2007) et Bozel Tincave (Rey, 2007). A l'exception de Tincave, les rituels sont remarquablement proches et se rattachent sans conteste à la tradition «Chamblandes», fortement présente en Valais et sur le pourtour lémanique, plus ponctuellement représentée dans les avant-pays savoyards, en Ligurie et vers le nord-ouest de la France (Chambon, 2007). Plus mal documentés, les autres sites (Aigueblanche, BourgSaint-Maurice) semblent relever des mêmes influences (Rey et Treffort, à paraître).

Dans les nécropoles lémaniques et valaisannes, la plupart des inhumations remontent aux deux derniers tiers du $\mathrm{V}^{\mathrm{e}}$ millénaire av. J.-C. L'utilisation de ces sites semble s'interrompre après 3800 av. J.-C. en Valais (Moinat et al., 2007), tandis que quelques cas ponctuels plus tardifs se rencontrent dans le bassin lémanique (Moinat, 2007). Dans notre zone d'étude les éléments de chronologie sont encore minces. Plusieurs datations ont été réalisées sur les tombes d'Aime Le Replat (Gély, 2005 ; figure 7B). Après une première analyse aujourd'hui rejetée, une nouvelle série de dates a livré des résultats centrés sur l'intervalle 4500-4300 av. J.-C., tout à fait cohérents avec la chronologie des sites suisses. Par ailleurs trois des nécropoles valdôtaines (SaintNicolas, Sarre et Vollein) ont fourni des bracelets en coquilles marines identiques aux exemplaires issus des tombes valaisannes, et pourraient donc se situer dans la même fourchette chronologique (Moinat et al., 2007).

A Chiomonte, F. Fedele considère que les inhumations sont contemporaines de l'habitat voisin, daté du premier tiers du IV ${ }^{\mathrm{e}}$ millénaire av. J.-C. (Fedele, 2007). Cette hypothèse qui ne repose sur aucun lien stratigraphique, pourrait impliquer une certaine perduration du rituel en Val de Suse. Le mobilier céramique de l'habitat contient cependant quelques décors gravés de type Chasséen ancien, qui permettent d'étendre l'occupation du site aux derniers siècles du $\mathrm{V}^{\mathrm{e}}$ millénaire av. J.-C.

Ainsi dans la zone considérée, si la disparition des sépultures de type «Chamblandes» ne peut être considérée comme parfaitement datée, la majorité des sites se rattache vraisemblablement au même intervalle que les nécropoles valaisannes, soit 4700-3800 av. J.-C. verts à Aime (figure 5) sont constitués de petites perles cylindriques en lignites qui trouvent des comparaisons préférentiellement dans les sites du bassin lémanique, et de deux pendeloques en roche verte en forme de goutte (Gély et al., 1991). A Chiomonte, le mobilier funéraire très pauvre n'est pas décrit. Sur ces bases fragiles, il est difficile pour l'instant de rattacher les nécropoles de Tarentaise et du Val de Suse à l'un de ces deux ensembles. La rareté des autres composantes du mobilier funéraire rend délicate l'identification culturelle des inhumés. A Aime, la sépulture 6 contenait 4 individus, dont deux, datés entre 4550 et 4230 av. J.-C., étaient accompagnés de perles cylindriques en lignite et de deux armatures de flèche (figure 5) : une petite pointe triangulaire à base concave en quartz hyalin qui trouve des comparaisons à la fois sur le plateau suisse et en Italie du nord, et une armature foliacée en silex blond de forme losangique dotée d'une légère constriction de la partie proximale, qui renvoie aux productions de la culture italienne des Vases à Bouches carrées (VBQ).

Dans les Alpes, le lien entre le rite «Chamblandes » et les zones de production de haches polies en roche verte a été plusieurs fois souligné (Bocquet, 1997 ; Gallay, 2000 ; Thirault, 2004 a). La diffusion assez large vers le nord-ouest de la France de sépultures apparentées aux «Chamblandes » semble d'ailleurs suivre les axes préférentiels de diffusion des haches en roches alpines.

La tombe fouillée à Bozel Tincave se distingue nettement des précédentes : implanté sur un versant raide, un coffre allongé contenait un individu non replié, déposé sur le dos, tête au sud, accompagné d'une petite lame en silex. Une datation ${ }^{14} \mathrm{C}$ place cette inhumation entre 3650 et 3130 av. J.-C. avec un maximum de probabilité centré sur le $34^{\mathrm{e}}$ siècle av. J.-C. (Rey, 2007). Tincave constitue ainsi le seul site funéraire de la fin du NM2 documenté dans notre zone d'étude. La rareté générale des données pour cette période limite les comparaisons. En contexte alpin, la tombe sans mobilier de Sion La Gillière est placée en stratigraphie entre le NM2 et le Néolithique final (Baudais et Schmidt, 1995). En dehors des Alpes quelques comparaisons imparfaites existent en Suisse orientale et dans la région de Schaffhouse (Moinat et Stöckli, 1995). 


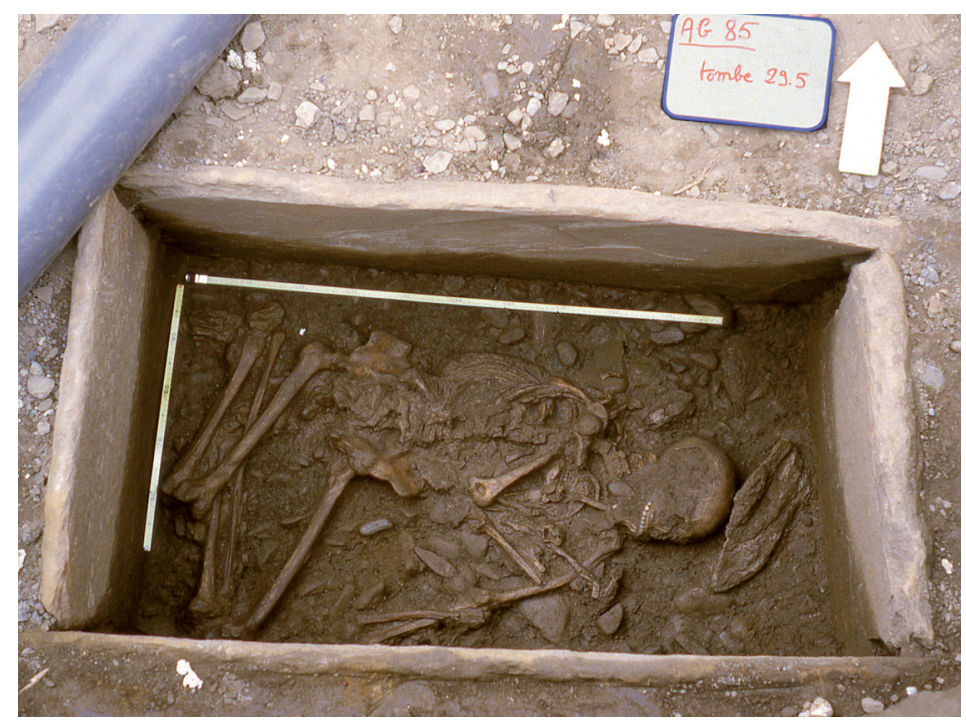

Figure 4 - Aime, Le Replat sépulture 1

(photo Pierre Ougier-Simonin).

Maddalena à Chiomonte (Bertone et Fozzati, 2002), fouillé dans le cadre d'une opération d'archéologie préventive dont la publication reste encore incomplète.

La plupart des implantations sont situées le long du réseau hydrographique principal dans la partie inférieure des versants, à proximité du fond de vallée. Deux sites seulement s'en éloignent nettement et se trouvent dans des secteurs d'alpages, sur ou à proximité d'itinéraires menant à des cols transalpins secondaires : Bessans La Teha à $2250 \mathrm{~m}$ (Thirault, 2006) et BSM Beaupré à $2130 \mathrm{~m}$ (Rey et al., 2008). Vraisemblablement liée à l'exploitation des ressources naturelles ou au franchissement

Durant le Néolithique moyen, les sites funéraires montrent donc une répartition chronologique inégale. La rareté des sites du NM2 pourrait s'expliquer par un déplacement vers des contextes moins investigués (versants, cônes torrentiels) ou par le développement de pratiques moins détectables. La récente mise en évidence, d'inhumations en fosse et d'incinérations sur les sites de Saint Léonard et de Bramois (Mariéthoz, 2007), à partir de 4300 av. J.-C., pourrait aller dans ce sens.

Après 3400/3300 av. J.-C. le début du Néolithique final est marqué par un profond renouvellement des pratiques funéraires dans les Alpes comme dans les régions périphériques. On trouve alors des sépultures collectives en grotte, en hypogée ou en caveau mégalithique, accompagnées ou non de la pratique de l'incinération. Des nécropoles de sépultures individuelles en fosse se développent au même moment dans la plaine du Pô (Remedello, Spilamberto).

\section{2 - Les sites d'habitat}

Dans une acceptation très large du terme, seize habitats sont connus dans notre espace d'étude, sans préjuger du caractère ponctuel, saisonnier ou permanent des occupations (figure 1 et tableau 1). Ils se répartissent surtout dans la partie médiane de la Tarentaise, en haute Maurienne et vers le débouché des vallées italiennes. Rares sont les sites étudiés sur des superficies importantes. La grotte des Balmes a connu une longue fouille programmée dont la publication est en cours (Vital et al., 2008). Le site de Bozel Chenet des Pierres est fouillé depuis 1999 (Rey, 2006) mais les résultats sont en grande partie inédits. Des sondages ont été réalisés à Bessans Rocher du Château (Thirault, 2004b) et sur plusieurs sites de Tarentaise : Bourg-Saint-Maurice (BSM) Châtelard (Rey et Treffort, à paraître), Bellentre Les Vignettes (Rey, à paraître ; figure 6) et Aime Dos de Borgaz (Rey, 2007). Les sites italiens ont fait l'objet d'interventions assez ponctuelles à l'exception de La de la crête, leur fonction reste difficile à préciser pour l'instant.

La répartition altitudinale des autres habitats montre une dichotomie entre les quatre sites mauriennais localisés entre 1000 et $1750 \mathrm{~m}$ d'altitude, et les implantations des autres vallées qui ne dépassent pas les $1000 \mathrm{~m}$ d'altitude, soit une cote maximale comparable aux données valaisannes (Curdy, 2007). La particularité de la Maurienne résulte surtout de l'histoire de la recherche : plusieurs archéologues ont vécu ou travaillé en haute Maurienne dans les trente dernières années. On remarquera par ailleurs que les sites mauriennais sont installés dans des contextes d'abri de pied de paroi (Rocher des Amoureux, Le Châtel et Rocher du Château) ou de grotte (Les Balmes à Sollières) qui ne se rencontrent pas parmi les sites implantés plus bas en altitude. Ils reflètent des occupations peu intenses au cours du NM, et pourraient être liés à des activités spécialisées (élevage, circulations, parcours de chasse, etc.). La quasi-totalité des dix autres habitats se caractérise par une implantation sur ou à proximité immédiate d'une position dominante, plus ou moins difficile d'accès et offrant un bon aperçu sur les axes de circulation. Seul Montalto Dora se distingue par son contexte de terrasse alluviale.

A l'exception des sites mauriennais, on retrouve une dispersion altitudinale assez comparable à ce que l'on connaît en Valais (Curdy, 2007). Les contextes d'implantation sont par contre moins diversifiés dans notre zone d'étude. Les habitats sur cône torrentiel y sont inconnus alors qu'ils sont bien représentés en Valais, où des complémentarités fonctionnelles avec les sites de hauteur ont été proposées (Gallay, 2000).

La documentation de la culture matérielle est pauvre et inégale. D'après les formes et décors des céramiques, les premières occupations assez discrètes interviennent vers le $46^{\mathrm{e}}$ siècle av. J.-C. sur deux sites à défenses naturelles qui présentent la remarquable particularité d'être implantés en pied d'ubac, et qui paraissent impossible à occuper de manière permanente : Aime Dos de Borgaz et Bozel Chenet des Pierres. 


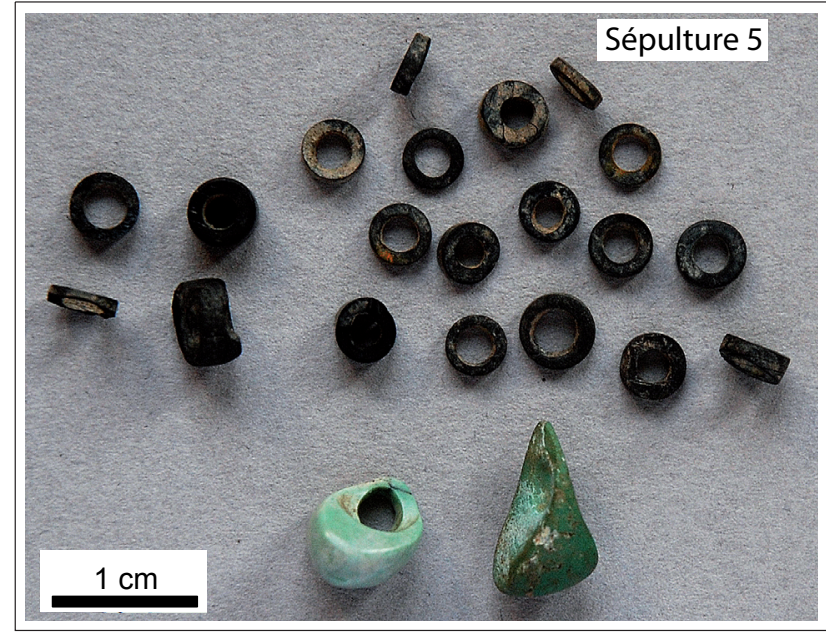

Figure 5 - Aime Le Replat : mobilier funéraire.
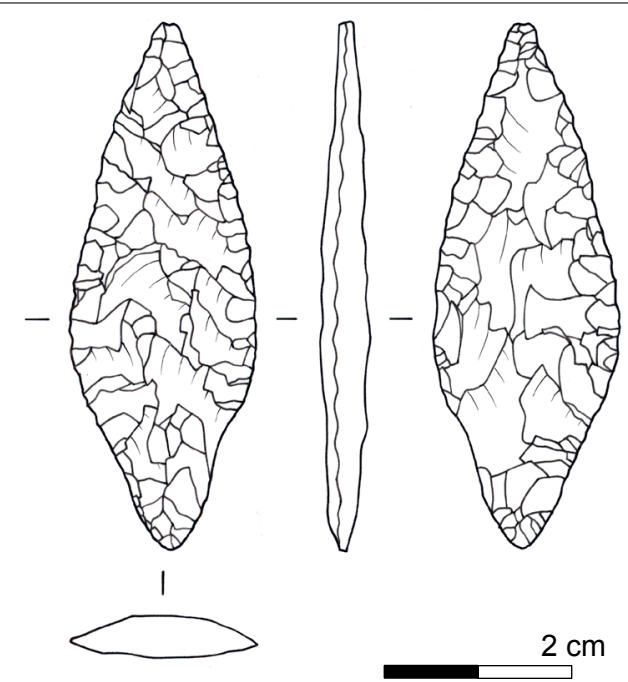

Sépulture 6

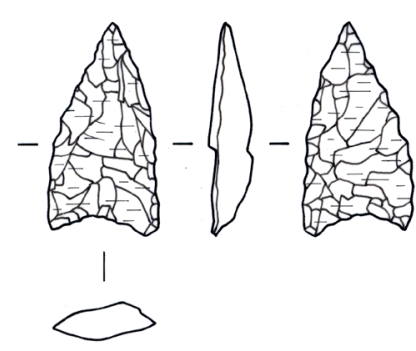

d'étude se retrouve assez largement dans les Alpes du nord française. Le site de Bessans fournit cependant plusieurs dates ${ }^{14} \mathrm{C}$ couvrant les deux derniers siècles $\mathrm{du} \mathrm{V}^{\mathrm{e}}$ millénaire av. J.-C. (Thirault, in Marguet et al., 2008), mais leur précision souffre de la présence d'un petit plateau de la courbe de calibration entre 4250 et 4050 cal BC. A Montaldo Dora, quelques tessons d'affinités Proto-lagozza (Venturino-Gambari, 2000) sont peut-être à peine plus récents.

$\mathrm{Au}$ début du $\mathrm{IV}^{\mathrm{e}}$ millénaire av. J.-C. des groupes régionaux (Saint-Léonard, Lagozza, Breno) s'individualisent dans les Alpes centrales et la plaine padane. Dans notre zone d'étude trois des habitats occupés précédemment sont réinvestis au cours du premier

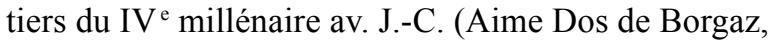
Bozel et peut-être le Châtelard de BSM), et de nouvelles implantations apparaissent à Saint-Pierre Châtelet, Chiomonte et Sollières Les Balmes. Si l'on excepte le cas particulier de la grotte des Balmes, les sites du début du IV ${ }^{\mathrm{e}}$ millénaire av. J.-C. semblent présenter un lien plus évident et plus systématique avec les positions dominantes et les aspects défensifs. Un phénomène comparable mais d'une toute autre ampleur s'observe durant la même période dans le Jura et dans les avantspays savoyards en concomitance avec le premier essor important de l'occupation des rives littorales (Marguet et Rey, 2007).

Largement documentée à Chiomonte et Bozel, la culture matérielle soulève des problèmes d'interpréta- 
tion. Il n'est pas possible actuellement d'individualiser des ensembles en succession stratigraphique, alors que les séries céramiques témoignent d'influences très variées, mais jamais intégralement représentées. Si des caractères assez nombreux évoquent le SaintLéonard et les faciès récents du Chasséen rhodanien, d'autres renvoient à la Lagozza, au Néolithique Moyen Bourguignon (NMB), au VBQ III, voire même à Breno. Cette diversité peut être liée aux modalités d'occupations (ponctuelles ou saisonnières), qui pourraient différer de la période précédente, ou à des particularités de statut ou de fonction. A Chiomonte, dans la nécropole proche de l'habitat, des structures particulières (alignement de pierres dressées, aire de combustion) ont été interprétées comme les vestiges d'une aire cérémonielle (Fedele, 2007). A Bozel les couches du NM1 ont livré une plaque de schiste façonnée en demi-cercle ornée de plusieurs cupules, alors que les dépôts du NM2 contenaient de petits objets en schiste dépourvus de fonction utilitaire (Rey, 2006). Par ailleurs une stèle trapézoïdale attribuée au $\mathrm{V}^{\mathrm{e}}$ millénaire av. J.-C. a été découverte dans les niveaux remaniés. Ces quelques éléments pourraient suggérer un rôle particulier de ce site dans l'organisation territoriale. L'hypothèse d'un lieu de rencontre et d'échange entre des populations diverses a été proposée pour Chiomonte (Bertone et Fozzati, 2002). Elle pourrait expliquer la présence d'un grand nombre d'éléments exogènes au sein du mobilier. Les séries céramiques seraient alors davantage représentatives du fonctionnement des réseaux d'échanges que de la culture matérielle locale. A Chiomonte et Bozel, de nombreux vases présentent cependant des originalités ou des caractères métis qui suggèrent une production locale, et constituent une bonne indication en faveur d'un peuplement intra alpin stable.

Les autres données sont nettement plus ponctuelles. À Aime un décor de cannelures verticales apparenté au Saint-Léonard et une barrette rectangulaire multiforée proviennent d'un horizon de réduction (Rey, 2007) ; aux Vignettes (figure 6) quelques fragments de marmites à profil légèrement sinueux et mamelons sous le bord évoquent le Cortaillod (Rey, à paraître). Quelques éléments de préhension perforés issus de Saint-Pierre près d'Aoste (Mezzena, 1997) suggèrent des influences chasséennes.

L'appréciation de la chronologie des occupations dépend en partie de l'intervalle retenu pour le SaintLéonard. A. Winiger (1994) a proposé de le placer entre 3700 et 3300 av. J.-C. Il pourrait cependant démarrer plus tôt d'après les premiers résultats de la fouille de sauvetage en cours sur le site éponyme (Mariethoz, 2007), ce qui étend la période potentielle d'occupation de Bozel et Chiomonte au-delà de la majorité des dates ${ }^{14} \mathrm{C}$ disponibles (figure $7 \mathrm{~A}$ ). À Bozel la plupart des autres composantes de la culture matérielle semblent indiquer un terminus de l'occupation vers 3500 av. J.-C.

Quatre habitats ont fait l'objet de séries de datations couvrant tout le Néolithique moyen : Aime Dos de Borgaz, Bozel, Bessans Rocher du Château et Chiomonte (figure 7A). Des dates uniques sont disponibles sur un niveau d'occupation de la grotte des Balmes à Sollières, et sur une structure en creux de Bellentre Les Vignettes. La date de BSM Côte du Moulin 2 documente un épisode d'incendie vraisemblablement lié à une fréquentation humaine.

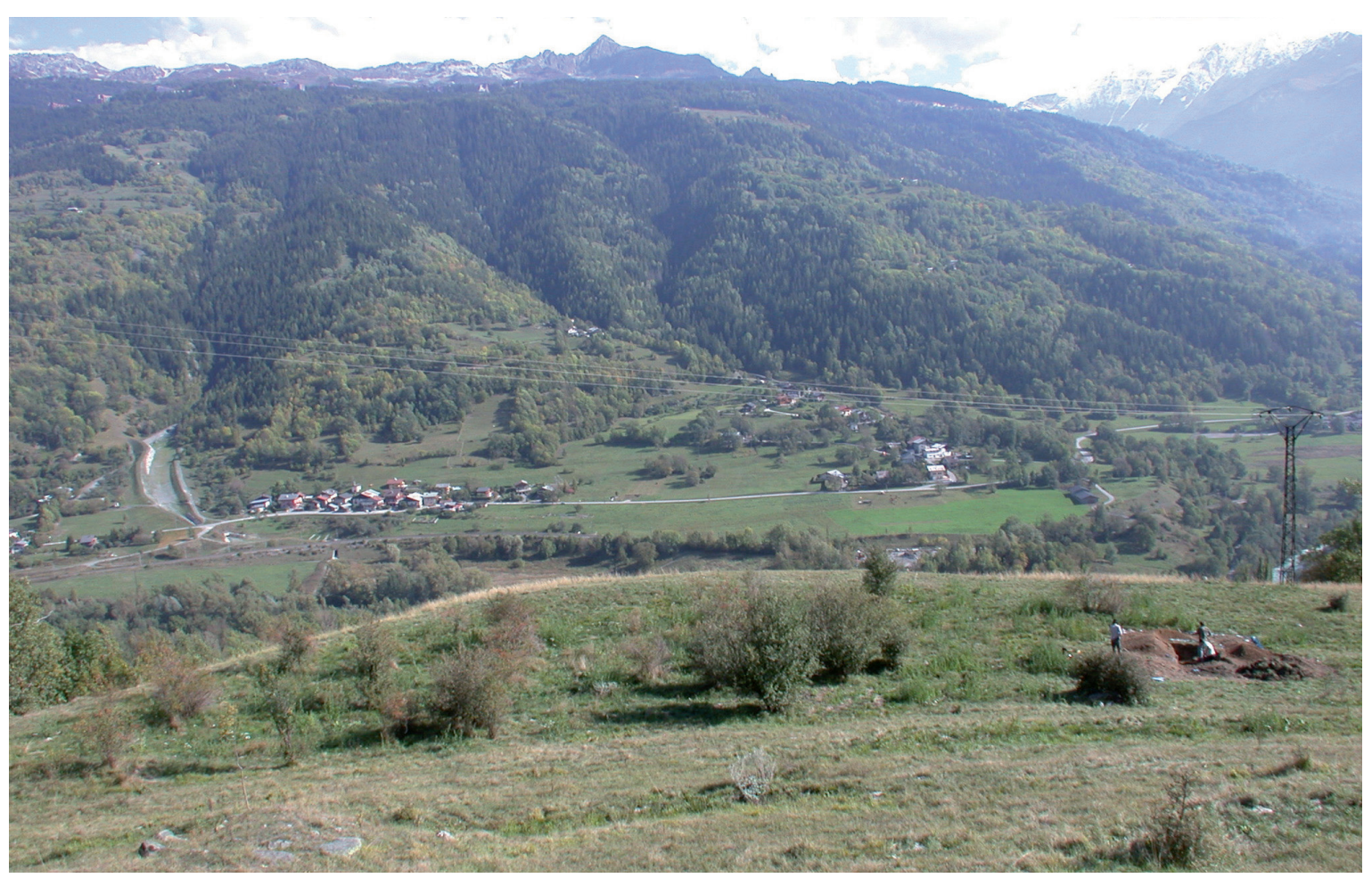

Figure 6 - Vue générale du site des Vignettes à Bellentre (photo Pierre-Jérôme Rey). 
Ces datations ne peuvent prétendre représenter l'ensemble des durées d'occupation comme l'illustrent les exemples du Chenet des Pierres et du Dos de Borgaz (figure 7A). Mais leur regroupement dans les intervalles 4500-4000 av. J.-C. et 3800-3600 av. J.-C. (figure $7 \mathrm{~A}, \mathrm{~B}$ ) paraît néanmoins confirmer la raréfaction des occupations perceptible dans les données matérielles, à la transition $\mathrm{V}^{\mathrm{e}}-\mathrm{IV}^{\mathrm{e}}$ millénaire av. J.-C. Ce phénomène pourrait démarrer dès le $42^{\mathrm{e}}$ siècle sur la plupart des sites, à l'exception de Bessans qui livre des dates ${ }^{14} \mathrm{C}$ centrées sur la fin $\mathrm{du} \mathrm{V}^{\mathrm{e}}$ millénaire av. J.-C., de Chiomonte d'où proviennent quelques décors gravés de type Chasséen ancien et peut-être de Montalto Dora (indices Proto-Lagozza mal calés entre
4300 et 3800 av. J.-C.). Toutefois, cette diminution des occupations peut recevoir d'autres explications qu'une baisse du peuplement : troncatures sédimentaires ou délocalisation des sites vers des milieux peu étudiés (cônes torrentiels, replats de versant).

Après 3400/3300 av. J.-C. la culture matérielle du début du Néolithique final témoigne d'un profond changement. Dans les Alpes et le Jura, on observe au cours des derniers siècles du IV ${ }^{\mathrm{e}}$ millénaire av. J.-C. un balancement entre des influences lointaines issues successivement du Horgen nord oriental puis du Ferrières méridional. Dans notre zone d'étude seule la grotte des Balmes à Sollières documente cette évolution de manière détaillée.

\section{II - Circulations, ÉChanges eT Économie de Subsistance}

L'étude des circulations de matières premières révèle le rôle majeur de l'exploitation des ressources naturelles dans les premières fréquentations des Alpes. Le fait le plus important semble la mise en place dès le Néolithique ancien des grands réseaux de diffusion de lames polies en éclogite et jadéite d'origine piémontaise. Elles circulent sous forme de deux productions très différentes : des biens de prestige non fonctionnels de taille et de facture exceptionnelles, intégrés dans des systèmes d'échanges compétitifs qui diffusent très loin vers l'ouest et le nord de l'Europe, et des lames fonctionnelles plus petites, utilisées pour les activités quotidiennes dans le bassin du Rhône et ses périphéries (Pétrequin et al., 2002 ; Thirault, 2004 a).

Ces deux productions présentent des évolutions chronologiques divergentes. La diffusion des biens de prestige est maximale au NM1 et se contracte très fortement vers 4100 av. J.-C. avant de cesser vers 3800 av. J.-C. ; tandis que la diffusion des lames fonctionelles en éclogite atteint son maximum au NM2 dans le bassin du Rhône avant de décliner au Néolithique final. Par les volumes mis en jeu, par leur extension géographique très large et par la durée de leur fonctionnement, les réseaux de circulation des roches vertes peuvent être considérés comme l'un des principaux éléments structurant des premiers peuplements alpins (Bocquet 1997 ; Thirault, 2004 a).

Sans atteindre la même envergure, d'autres circulations se développent sur de longues distances au Néolithique moyen (Beeching, 2003). Le silex issu des massifs calcaires est présent en quantité importante sur les sites bien documentés de Chiomonte, Bozel et Bessans, mais l'analyse des provenances reste à faire. On sait que les silex bédouliens du Vaucluse diffusent à large échelle parallèlement à la rapide expansion des influences chasséennes dans les derniers siècles du $\mathrm{V}^{\mathrm{e}}$ millénaire av. J.-C. A l'inverse, le quartz hyalin couramment utilisé dans les Alpes, apparaît fréquemment en vallée du Rhône au NM, sous forme d'un nombre limité de produits de très bonne facture. Cette importance des circulations au NM pourrait être un élément d'explication de l'apparent choix préférentiel des positions défensives ou dominantes.

Dans notre zone d'étude l'économie de subsistance reste assez difficile à appréhender, et il est nécessaire d'examiner brièvement la situation des régions périphériques. En Valais d'après des résultats issus d'un nombre limité de sites d'altitude assez basse, une économie pleinement agro-pastorale se maintient durant tout le NM (Gallay, 2000). En Italie septentrionale l'élevage caprin prend de l'importance au NM1 et le NM2 serait caractérisé par une mobilité nettement accrue et une économie essentiellement pastorale (Barfield et al., 2003).

En moyenne vallée du Rhône, le NM voit la longue évolution d'un système dans lequel le pastoralisme semble tenir la place principale. Dans la plaine alluviale et les piémonts, la végétation est profondément modifiée par le développement de pâtures arborées produisant un paysage qui se rapproche de la Dehesa espagnole actuelle (Delhon et al., 2008). La durée et l'intensité de ce type particulier d'exploitation du territoire se traduisent par le développement d'hori-

Figure 7 (ci-contre) - Synthèse des données archéologiques et climatiques.

$A:$ datations disponibles pour les sites d'habitats des vallées étudiées (en gris : dates à écarter);

$B:$ datations disponibles pour les sites funéraires des vallées étudiées, confrontées aux variations de la courbe de calibration;

$C$ : évolutions des pratiques funéraires en périphérie de la zone d'étude ;

$D$ : cadre chrono-culturel dans les régions voisines les mieux documentées ;

E : principaux épisodes de changements culturelles rapides dans les Alpes ;

$F$ : marqueurs des fluctuations climatiques. 


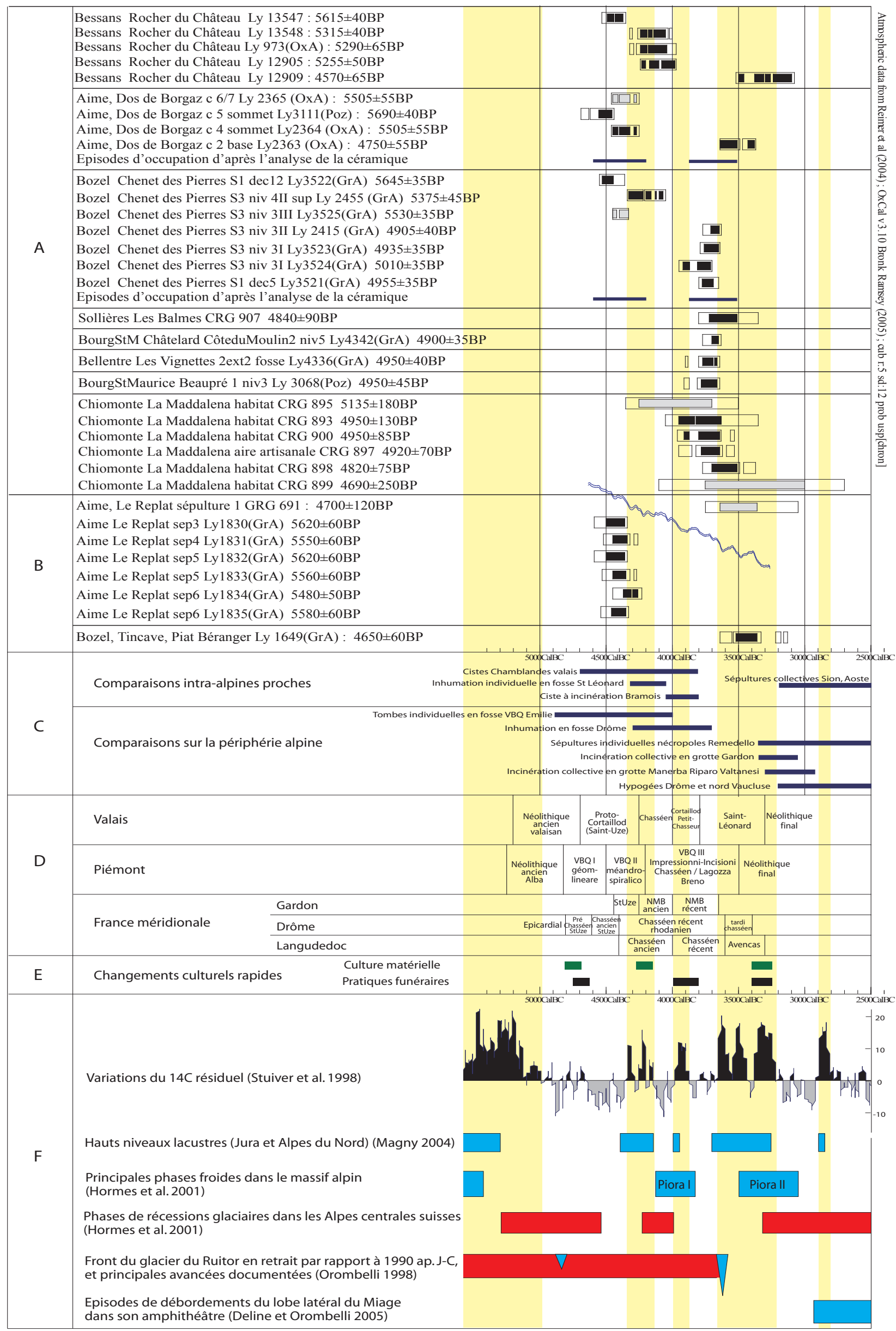


zons sédimentaires spécifiques : des sols bruns noirs proches des chernozems. Par ailleurs des cavités contenant d'épaisses accumulations de fumiers ont été mises en évidence de la plaine jusqu'aux contreforts des massifs préalpins et ardéchois (Brochier et Beeching, 2006). Ces grottes-bergeries témoignent de la mise en place d'un système de remues plaine / montagne. Les sites de la Grande Rivoire (Isère, $650 \mathrm{~m}$ ) et de la Balme de Thuy (Haute-Savoie) montrent l'extension de ces pratiques sur la périphérie des massifs interne, dès le début du NM (Nicod et al., 2008). La grotte des Balmes à Sollières constitue le seul cas de grotte bergerie connue dans les vallées alpines internes, mais cet usage ne semble s'y développer qu'à partir du Néolithique final (Vital et al., 2008).

Aucune structure pastorale n'est connue dans les Alpes occidentales avant le Néolithique final / Bronze ancien (Mocci et al., 2008 ; Rey et al., 2008).

Les données polliniques disponibles dans les Alpes françaises montrent un impact humain qui reste discret au Néolithique (De Beaulieu et Goeury, 2004). A quelques exceptions localisées en Champsaur et dans le Buech, les impacts anthropiques demeurent cantonnés en dessous de $1200 \mathrm{~m}$ d'altitude. Si les atteintes au milieu paraissent se multiplier un peu au Néolithique final, il faut attendre le Bronze final/Hallstatt ancien pour voir se développer largement les défrichements dans les zones d'altitude propices à l'estive des troupeaux. Il est difficile de préciser si l'absence d'enregis- trement durant le NM traduit une absence d'activités ou si les atteintes au milieu sont trop réduites, trop dispersées, ou trop cantonnées dans les basses altitudes peu concernées par les carottages polliniques, pour être actuellement bien perçues.

Dans notre espace d'étude les données sont parcellaires. À Bozel le maintien d'une importance de la chasse semble transparaître dans la proportion élevée des armatures au sein des outillages taillés (étude en cours par T. Perrin). Mais la difficulté à distinguer caprinés sauvages et domestiques dans un corpus très fragmenté nuit à une bonne perception du rapport chasse/élevage. À Chiomonte, bien que le bestiaire domestique dominé par les caprinés ne soit pas négligeable, la faune chassée reste majoritaire (Bertone et Fozzati, 2002).

Différentes espèces de céréales sont attestées à Bozel et Sollières dans les niveaux du NM sans que l'on puisse pour l'instant démontrer leur culture sur place (Martin et al., 2008). Plusieurs meules en pierres ont été retrouvées sur ces deux sites, mais également au Dos de Borgaz et à Bellentre.

Pour l'instant l'économie de subsistance des communautés néolithiques nous échappe donc très largement. La part respective des pratiques agricoles, pastorales et cynégétiques ne peut être évaluée, alors qu'il s'agit d'un point crucial pour estimer la fragilité des sociétés, et pour identifier les périodes de changement (Arbogast et al., 1995).

\section{III - Fluctuations CLIMATIQUES ET CHRONOLOGIE DES ÉVOLUTIONS SOCIALES}

Les évolutions climatiques holocènes sont décrites de manière indirecte par différents signaux (figure $7 \mathrm{~F}$ ). Les variations $d u{ }^{14} \mathrm{C}$ résiduel atmosphérique qui constituent un marqueur indirect de l'activité solaire (Stuiver et al., 1998), et les fluctuations des lacs sub-alpins et jurassiens (Magny, 2004) seront utilisées en priorité compte tenu de leur excellente précision chronologique. En contexte alpin les fluctuations du détritisme dans le lac du Bourget (Arnaud et al., 2005, 2008) et les fluctuations des glaciers suisses (Hormes et al., 2001) apportent des éléments d'appréciation complémentaires. Dans notre zone d'étude, les données obtenues sur les glaciers du Ruitor (Orombelli, 1998) et du Miage (Deline et Orombelli, 2005) fournissent des indications précieuses pour le Néolithique moyen.

L'apparition des premiers sites au début du Néolithique moyen 1, ainsi que la croissance des circulations de roche verte, interviennent dans une période de climat plutôt favorable et stable.

L'expansion chasséenne sur la périphérie des Alpes autour de 4200 av. J.-C. (Nicod, à paraître) ainsi que l'effondrement quasi complet du système d'échange compétitif basé sur les grandes lames polies, prennent place vers 4100 av. J.-C., au cours d'une péjoration cli- matique marquée par trois pics rapprochés de la courbe $\mathrm{du}{ }^{14} \mathrm{C}$ atmosphérique, par le début d'une longue période de hauts niveaux lacustres, et par une phase d'avancée glaciaire dans les Alpes suisses (figure $7 \mathrm{~F}$ ).

La rareté des données archéologiques dans les deux derniers siècles du $\mathrm{V}^{\mathrm{e}}$ millénaire av. J.-C. intervient alors que la courbe $\mathrm{du}{ }^{14} \mathrm{C}$ résiduel atmosphérique redevient négative, les niveaux lacustres diminuent et les glaciers des Alpes centrales suisses sont en retrait. Cependant la phase froide Piora I pourrait débuter dès 4150 av. J.-C. (Hormes et al., 2001).

Dans les deux premiers siècles du IV e millénaire av. J.-C., le rituel «Chamblandes» s'efface progressivement et les échanges compétitifs basés sur les grandes lames polies disparaissent alors que tous les signaux climatiques indiquent une dégradation centrée sur le $40^{\mathrm{e}}$ siècle av. J.-C., qui pourrait marquer le maximum de Piora I. Entre 3860 et 3650 av. J.-C. un nouvel essor de l'anthropisation se développe alors que les indicateurs climatiques témoignent à nouveau d'une amélioration.

Ensuite les données archéologiques se raréfient considérablement dans notre zone d'étude comme dans l'ensemble des Alpes occidentales et de leurs périphéries. De profonds bouleversements interviennent dans 
la culture matérielle : les groupes locaux cèdent la place à des influences issues de régions péri-alpines. Des rites funéraires nouveaux apparaissent alors que les réseaux de diffusion de l'outillage en roche verte se replient sur le bassin du Rhône moyen. Des innovations techniques (araire et complexe de la traction) et des mutations sociales (évolution du statut masculin) interviennent vers 3500 av. J.-C. Dans le même temps la remontée des niveaux des lacs à partir de 3700 et l'inversion de la courbe $d u{ }^{14} \mathrm{C}$ résiduel atmosphérique après 3650 av. J.-C. marquent le début d'une dégradation importante, qui dure jusque vers 3230 av. J.-C. et correspond avec un léger décalage à la phase froide Piora II.

Cette péjoration plus longue que les deux précédentes pourrait également avoir été d'une intensité supérieure. Magny et Hass (2004) ont montré qu'elle correspondait à un phénomène mondial qu'ils considèrent comme le début du Néoglaciaire : une succession rapprochée de phases froides de forte amplitude qui marque la seconde moitié de l'Holocène, sous l'influence d'une lente décroissance de l'insolation solaire dans l'hémisphère nord.

Alors que les oscillations des $43^{\mathrm{e}}$ et $40^{\mathrm{e}}$ siècle av. J.-C. n'ont aucune traduction dans la courbe du détri- tisme dans le lac du Bourget (Arnaud et al., 2008), celle-ci montre un net accroissement dans le second tiers $\mathrm{du} \mathrm{IV}^{\mathrm{e}}$ millénaire av. J.-C. parfaitement synchrone avec la transgression lacustre. Vers 3650 av. J.-C. le glacier du Ruitor dépasse son front de 1990 ap. J.-C. pour la première fois depuis le début de l'Holocène. Bien qu'intervenu un peu plus tardivement, le premier des débordements du Miage dans son amphithéâtre est probablement à relier au même événement, en raison du temps nécessaire à la mise en place de sa couverture sédimentaire (Deline et Orombelli, 2005).

Orombelli soutient la même hypothèse en s'appuyant sur les fluctuations du Ruitor qui est toujours resté en avant de sont front de 1990 ap. J.-C. depuis 3650 av. J.-C. (Orombelli, 1998), et sur la conservation du corps momifié d'Ötzi dans les Alpes centrales, qui suppose un enfouissement rapide suivi du maintien d'un englacement quasi ininterrompu depuis 3300-3000 av. J.-C. jusqu'à l'actuel (Baroni et Orombelli, 1996).

Le début du Néoglaciaire est cependant discuté (Deline et Le Roy, 2008), d'autres auteurs proposant la phase froide de Loëbben pendant le Bronze moyen, ou de Göschenen I au début du Premier Âge du Fer, mais les remarques précédentes sur l'extension de l'englacement alpin ne sont pas remises en cause.

\begin{tabular}{|c|c|c|c|c|c|}
\hline $\mathbf{N}^{\circ}$ de site & Commune et nom du site & Vallée & Altitude & $\begin{array}{c}\text { Contexte } \\
\text { d'implantation }\end{array}$ & Type de site \\
\hline 1 & Aigueblanche Bellecombe & Tarentaise & 470 & cône torrentiel & funéraire \\
\hline 2 & Aime Dos de Borgaz & Tarentaise & 677 & site hauteur & habitat \\
\hline 3 & Aime Le Replat & Tarentaise & 663 & alluvial & funéraire \\
\hline 4 & Aime Saint-Sigismond & Tarentaise & 720 & site hauteur & habitat \\
\hline 5 & Bellentre Les Vignettes & Tarentaise & 895 & site hauteur & habitat \\
\hline 6 & Bourg-Saint-Maurice Le Châtelard & Tarentaise & 882 & site hauteur & funéraire et habitat \\
\hline 7 & Bourg-Saint-Maurice Beaupré & Tarentaise & 2130 & plateau d'altitude & habitat \\
\hline 8 & Bozel Chenet des Pierres & Tarentaise & 945 & site hauteur & habitat \\
\hline 9 & Bozel Tincave & Tarentaise & 1215 & versant & funéraire \\
\hline 10 & Villarodin Rocher des Amoureux & Maurienne & 1195 & replat & habitat \\
\hline 11 & Sollières abri du Chatel & Maurienne & 1320 & abri sous roche & habitat \\
\hline 12 & Sollières grotte des Balmes & Maurienne & 1350 & grotte & habitat \\
\hline 13 & Bessans Rocher du Château & Maurienne & 1755 & terrasse alluviale & habitat \\
\hline 14 & Bessans La Teha & Maurienne & 2250 & versant & habitat \\
\hline 15 & Saint-Nicolas & vallée d'Aoste & 1196 & site de hauteur & funéraire \\
\hline 16 & Villeneuve Champrotard & vallée d'Aoste & 678 & terrasse alluviale & funéraire \\
\hline 17 & Saint-Pierre Châtelet & vallée d'Aoste & 812 & site de hauteur & habitat \\
\hline 18 & Sarre & vallée d'Aoste & 660 & $?$ & funéraire \\
\hline 19 & Quart Vollein & vallée d'Aoste & 912 & site de hauteur & funéraire \\
\hline 20 & Montjovet & vallée d'Aoste & 417 & replat & funéraire \\
\hline 21 & Montalto Dora & vallée d'Aoste & 270 & terrasse alluviale & habitat \\
\hline 22 & Pont Canavese Santa Maria & vallée de l'Orco & 540 & site de hauteur & habitat \\
\hline 23 & Chiomonte La Maddalena & Val de Suse & 730 & replat & funéraire et habitat \\
\hline 24 & Borgone di Susa San Valeriano & Val de Suse & 390 & site de hauteur & habitat \\
\hline
\end{tabular}

Tableau 1 - Inventaire des sites pris en compte. La numérotation correspond à la légende de la figure 1. 


\section{Conclusion}

Prises isolément, les données collectées sont peu significatives. Ainsi la dynamique du peuplement reste difficile à appréhender sur la base du nombre de sites connus. C'est la multiplication des changements synchrones (figure $7 \mathrm{E}$ ) qui peut faire sens en regard des évolutions climatiques (figure 7F).

Dans notre zone le Néolithique moyen peut être subdivisé en quatre phases : entre 4600 et 4200 av. J.-C. densification des occupations et des échanges, affirmation d'une identité culturelle autour des rites funéraires ; entre 4200 et 3900 av. J.-C. raréfaction des sites, perte d'identité culturelle, contraction ou changement des réseaux d'échange ; entre 3900 et 3600 av. J.-C. densification des sites, affirmation d'une identité culturelle basée sur les formes et décors céramiques, intensification des échanges ; entre 3600 et 3300 av. J.-C., grande raréfaction des occupations, contraction des échanges, changements culturels, techniques et sociaux.

Les phases d'affirmation d'une identité culturelle alpine interviennent au cours des deux plus longues périodes d'améliorations climatiques, alors que le nombre des sites connus est élevé. Une corrélation nette entre rupture dans l'évolution des sociétés et dégradation climatique est probable à la transition Néolithique moyen/Néolithique final. L'épisode Piora II semble coïncider avec une crise majeure dans l'évolution des sociétés néolithiques, particulièrement ressentie en montagne. Cette corrélation reste moins évidente pour les deux phases précédentes de dégradation car les changements paraissent moins synchrones entre eux et pourraient recevoir d'autres explications.
L'accessibilité des cols joue un rôle évident pour le fonctionnement des réseaux d'échanges, mais dans le cas des roches vertes par exemple, l'essor de productions concurrentes (minières de silex) et/ou l'épuisement des ressources en combustible dans les zones d'extraction, pourraient aussi expliquer les changements qui se développent vers 4100 av. J.-C.

La rareté des données dans des périodes de climat plutôt favorable, à la transition Néolithique ancien/Néolithique moyen et entre 4200 et 3900 av. J.-C., souligne le caractère non systématique de la corrélation entre évolutions sociales et fluctuations climatiques.

Les données archéologiques disponibles dans les Alpes nord-occidentales paraissent compatibles avec l'hypothèse d'une sensibilité importante des populations aux fluctuations climatiques durant le NM, ce qui rejoint les propositions avancées pour le Jura (Pétrequin et al., 2004). Dans les Alpes nord-occidentales durant le NM, cette sensibilité pourrait être croissante avec le temps en accord avec l'intensité croissante des dégradations climatiques. Dans ce milieu particulièrement sensible, les variations climatiques se traduisent vraisemblablement par des diminutions rapides et importantes du rendement des cultures et de la production fourragère. Ces conclusions soulignent les insuffisances des tentatives actuelles de modélisation du peuplement alpin, qui décrivent un investissement progressif des versants (Fedele, 1999 ; Curdy, 2007). Ils paraissent dotés d'une trop faible résolution géographique et chronologique, pour rendre compte d'une évolution qui semble non linéaire au Néolithique moyen.

\section{Remerciements}

Les données nouvelles sur la Tarentaise ont été acquises depuis 1999, dans le cadre de plusieurs opérations archéologiques programmées, grâce au soutien du Ministère de la Culture, du Département de la Savoie et du GDR Juralp. Mes remerciements vont également à Fabien Arnaud, Michel Magny et Pierre-Yves Nicod qui ont acceptés de relire cet article.

\section{BibLIOGRAPHIE}

Arbogast, R.-M., Magny M., PÉtrequin P., 1995. Expansions et déprises agricoles au Néolithique : populations, cultures céréalières et climat dans la combe d'Ain (Jura, France) de 3700 à 2500 av JC. In Van Der Leeuw S. dir. L'homme et la dégradation de l'environnement. Actes des $\mathrm{XV}^{\mathrm{e}}$ Rencontres Internationales d'Archéologie et d'Histoire d'Antibes. APDCA : Juan-Les-Pins, 19-41.

Arnaud F., Wessels M., Chapron E., Revel-Roland M., Desmet M., 2008. Signaux terrigènes dans les grands lacs subalpins ; un marqueur régional à haute résolution des conditions paléohydrologiques. In Magny M., Desmet M. et Mocci F. dir. Du climat à l'homme; dynamique holocène de l'environnement dans le Jura et les Alpes. Actes de la table ronde du GDR JURALP, Aix-en-Provence novembre 2007. Collection Edytem, 6, 77-88.
Arnaud F., Revel-Rolland M., Chapron E., Desmet M., TRIBOVILLARD N., 2005. 7200 years of Rhône river flooding activity recorded in Lake Le Bourget: A high resolution sediment record of NW Alps hydrology. The Holocene, $15-3,420-428$.

Barfield L-H., Bernabo Brea M., Maggi R., Pedrotti A., 2003. Processi di cambiamento culturale nel neolitico dell'italia settentrionale. Atti XXXV riunione scientifica IIPP, Castello di Lipari, Chiesa di S. Caterina 2-7 giugno 2000. Firenze : IIPP, II, 665-685.

Baroni C., Orombelli G., 1996. The Alpine "Iceman" and holocene climatic change. Quaternary Research, 46, 78-83.

Baudais D., Schmidt P.-Y., 1995. Le site de Sion La Gillière. In Gallay A. dir. Dans les Alpes à l'aube du métal ; Archéologie et bande dessinée. Sion : Musées Cantonaux du Valais, 96-102. 
BAZZANELla M., 1997. Les vases à ouverture carrée en Europe occidentale. In Constantin C., Mordant C., Simonin D. dir La culture de Cerny : nouvelle économie, nouvelle société au néolithique. Actes du $6^{\mathrm{e}}$ Colloque International de Nemours (9-11 mai 1994). Mém. Musée Préh. d'Île-deFrance, 6. Nemours : APRAIF, 557-57.

BEECHING A., 1999. Les premières étapes de circulation et de peuplement dans les Alpes françaises au Néolithique ; Apport de la céramique. In Beeching A. dir. Circulations et Identités culturelles alpines à la fin de la Préhistoire. Programme CIRCALP 1997-1998. Travaux du CAP Valence, 2, 427-479.

BeEching A., 2003. Mobilité et sociétés néolithiques dans les Alpes occidentales et la France méridionale. Prehistoria Alpina, 39, 175-187.

Berger J.-F., 2005. Sédiments, dynamique du peuplement et climat au Néolithique ancien. In Guilaine J. dir. Populations néolithiques et environnements. Paris : Errance, 153-212.

Bertone A., Fozzati L., dir. 2002. 6000 anni di storia sulle Alpi Occidentali ; La Maddalena di Chiomonte. Torino : Nautilus. 197 p. BocQuet A., 1997. Archéologie et peuplement des Alpes françaises du Nord au Néolithique et aux Âges des métaux. L'Anthropologie, 101(2), 291393.

Brochier J.-L., BeEching A., 2006. Grottes-bergeries, pastoralisme et mobilité dans les Alpes au Néolithique. In Jourdain-Annequin C. et Duclos J.-C. dir. Aux origines de la transhumance. Paris : Picard, 131-157.

Chambon P., 2007. Des Chamblandes au centre de la France? In Moinat P. et Chambon P. dir. Les cistes Chamblandes et la place des coffres dans les pratiques funéraires du Néolithique moyen occidental. Actes du colloque de Lausanne, 12 et 13 mai 2006. CAR 110, Mém. SPF XLIII, 75-89.

Curdy P., 2007. Prehistoric settlement in middle and high altitudes in the Upper Rhone Valley (Valais-Vaud, Switzerland) : A summary of twenty years of research. Prehistoria Alpina, 42, 99-108.

De Beaulieu J.-L., Goeury C., 2004. Les premiers signes de l'anthropisation dans les Alpes françaises d'après l'analyse pollinique. In Richard H. dir. Néolithisation précoce. Premières traces d'anthropisation du couvert végétal à partir des données polliniques. Collection Annales Littéraires de l'Université de Franche-Comté 777, série Environnement, sociétés et archéologie 7. Besançon : PU Franc-Comtoises, 163-171.

Delhon, C., Thiebault S., Berger J.-F., 2008 in press. Environment and landscape management during the Middle Neolithic in Southern France : Evidence for agro-sylvo-pastoral systems in the Middle Rhone Valley. Quaternary International.

Deline P., Orombelli G., 2005. Glacier fluctuations in the western Alps during the Neoglacial, as indicated by the Miage morainic amphitheatre (Mont Blanc massif, Italy). Boreas, 34(4), 456-467.

Deline P., Le Roy M., 2008. Fluctuations des glaciers des Alpes occidentales depuis 5000 ans : un état des connaissances. In Magny M., Desmet M. et Mocci F. dir. Du climat à l'homme ; dynamique holocène de l'environnement dans le Jura et les Alpes. Actes de la table ronde du GDR JURALP, Aix-en-Provence novembre 2007. Collection Edytem, 6, 13-28.
Fedele F., 1999. Peuplement et circulation des matériaux dans les Alpes occidentales du Mésolithique à l'Âge du Bronze. In Beeching A. dir. Circulations et Identités culturelles alpines à la fin de la Préhistoire. Programme CIRCALP 1997-1998. Travaux du CAP Valence 2, 331-358.

Fedele F., 2007. La nécropole de La Maddalena à Chiomonte, vallée de Suse (3900-3700 av. JC). In Moinat P. et Chambon P. dir. Les cistes Chamblandes et la place des coffres dans les pratiques funéraires du Néolithique moyen occidental. Actes du colloque de Lausanne, 12 et 13 mai 2006. CAR 110, Mém. SPF XLIII, 309-323.

Gallay A., avec coll. Nicod P.-Y., 2000. Le Néolithique dans les Alpes occidentales. In Boëtsch G. dir. Evolutions biologiques et culturelles en milieu alpin. Univ. d'été de la Méditerranée Vallouise 1999. Aix-en-Provence : Université, 17-38.

Gely B., 2005. Nouvelles datations des restes humains néolithiques de la nécropole du Replat à Aime (Savoie). Bilan Scientifique de la région Rhône-Alpes 2003. Lyon : DRAC Rhône-Alpes, 186.

Gely B., Ougier-Simonin P., Porte J-L., 1991. Fouilles de sauvetage d'une nécropole néolithique à Aime (Savoie). In Rites funéraires et sépultures. Dernières découvertes et études de synthèse, Actes du 6ème colloque international sur les Alpes dans l'Antiquité, Annecy, sept.1989. BEPA, II, 41-56.

Hormes A., Müller B.-U., Schlüchter C., 2001. The Alps with little ice: evidence for eight Holocene phases of reduced glacier extent in the Central Swiss Alps. Holocene $11 / 3,255-265$

Magny M., 2004. Holocene climate variability as reflected by mid-European lake-level fluctuations and its probable impact on prehistoric human settlements. Quaternary International, 113 (1), 65-79.

Magny M., HaAs J.-N., 2004. A major widespread climatic change around 5300 cal. yr BP at the time of the Alpine Iceman. Journal of Quaternary Science, 19 (5), 423-430.

Marguet A., Bintz P., Nicod P.-Y., Picavet R., Rey P.-J., Thirault E., 2008. Eléments pour une histoire du peuplement nord-alpin français entre 10000 et 2700 ans BP. In Magny M., Desmet M. et Mocci F. dir. Du climat à l'homme ; dynamique holocène de l'environnement dans le Jura et les Alpes. Actes de la table ronde du GDR JURALP, Aix-en-Provence novembre 2007. Collection Edytem, 6, 227-254.

Marguet A., Rey P.-J., 2007. Le Néolithique dans les lacs alpins français : un catalogue réactualisé. In Besse $\mathrm{M}$. dir. Sociétés néolithiques ; des faits archéologiques aux fonctionnements socio-économiques. Actes du $27 \mathrm{e}$ colloque Internéo, Neuchâtel 1-2 octobre 2005. Cahier d'Archéologie Romande 108, 379-406.

Mariethoz F., 2007. Variabilité des pratiques funéraires en Valais autour de 4000 av. JC : découvertes récente. In Moinat P. et Chambon P. dir. Les cistes Chamblandes et la place des coffres dans les pratiques funéraires du Néolithique moyen occidental. Actes du colloque de Lausanne, 12 et 13 mai 2006. CAR 110, Mém. SPF XLIII, 265-276.

Martin L., Jacomet S., Thiebault S., 2008. Plant economy during the Neolithic in a mountain context: the case of "le Chenet des Pierres" in the French Alps (BozelSavoie, France). Proceedings of the 14th Symposium of the International Work Group for Palaeoethnobotany, Kraków 2007. Vegetation History and Archaeobotany, 17, 1, 113-122. 
Mezzena F., 1997. La valle d'Aosta nel Neolitico et nell' Eneolitico. In La valle d'Aosta nel quadro della preistoria et protostoria dell' arco alpino centro-occidentale. Atti $\mathrm{XXXI}^{\mathrm{e}}$ Riunione Scientifica, Courmayeur 2-5 giugno 1994. Firenze : IIPP, 17-138

Mocci F., Walsch K., Richer S., Court-Picon M., Talon B., Tzortzis S., Palet-Martinez J.-M., Bressy C., 2008. Archéologie et paléo-environnement dans les Alpes méridionales françaises : hauts massifs de l'Argentièrois, du Champsaur et de l'Ubaye, Néolithique final - début de l'Antiquité. In Magny M., Desmet M. et Mocci F. dir. Du climat à l'homme ; dynamique holocène de l'environnement dans le Jura et les Alpes. Actes de la table ronde du GDR JURALP, Aix-en-Provence novembre 2007. Collection Edytem, 6, 253-272.

Moinat P., avec coll. Studer J., 2007. Cistes en pierres et coffres en bois, inhumations simples et dépôts complexes : un bilan des pratiques funéraires à Vidy (Lausanne, Vaud) et à Chamblandes (Pully, Vaud). In Moinat P. et Chambon P. dir. Les cistes Chamblandes et la place des coffres dans les pratiques funéraires du Néolithique moyen occidental. Actes du colloque de Lausanne, 12 et 13 mai 2006. CAR 110, Mém. SPF XLIII, 195-220.

Moinat P., Baudais D., Honneger M., Mariethoz F., 2007. De Bramois au Petit-Chasseur, une synthèse des pratiques funéraires en Valais central entre 4700 et 3800 av. JC. In Moinat P. et Chambon P. dir. Les cistes Chamblandes et la place des coffres dans les pratiques funéraires du Néolithique moyen occidental. Actes du colloque de Lausanne, 12 et 13 mai 2006. CAR 110, Mém. SPF XLIII, p. 297-308.

MoINAT P., STÖCKLI W.-E., 1995. Croyances et rites funéraires. In La Suisse du Paléolithique à l'aube du Moyen-Âge (SPM) vol. II ; Néolithique. Bâle : SSPA, 231-258.

Nicod P.-Y., Coutard C. (à paraître). Les céramiques du Néolithique moyen I (couches 52 à 48). In Voruz J.-L. et Perrin T. dir. Monographie de la grotte du Gardon, vol. 1.

Nicod P.-Y., Picavet R., Argant J., Brochier J.-L., Chaix L., Delhon C., Martin L., Moulin B., Thiebault S., 2008. La bergerie néolithique de la Grande Rivoire. In Jospin J.-P. et Favrie T. dir. Premiers bergers des Alpes de la Préhistoire à l'Antiquité. Musée Dauphinois : Infolio, 75-79.

Orombelli G., 1998. Le torbe del Rutor: una successione significativa per la storia olocenica dei ghiacciai e del clima delle Alpi. In Ghelardoni P. dir. Il clima e la storia del clima; Studi in onore di Mario Pinna. Memorie della Società Geografica Italiana, 55, 153-165.

Petrequin P., Cassen S., Croutsch C., Errera M., 2002. La valorisation sociale des longues haches dans l'Europe néolithique. In Guilaine J. dir. Matériaux, productions, circulations du Néolithique à 'Âge du Bronze. Paris : Errance, 67-98.

Petrequin P., Magny M., Bailly M., 2004. Habitat lacustre, densité de population et climat. L'exemple du Jura français. In Della Casa P., Trachsel M. dir. WES‘04 ; Wetland Economies and Societies. Proceedings of the International Conference, Zurich, 10-13 March 2004. Zurich : Musée suisse, Chronos Verlag (Collectio Archaeologica 3), 143168.

Rey P.-J., 2006. Le site du Chenet des Pierres aux Moulins de Bozel (Savoie, France) : une nouvelle séquence néolithique alpine. In Alpis Graia Archéologie sans frontières autour du col du Petit-Saint-Bernard, projet Interreg IIIA, seminario di chiusura Aoste 2-4 mars 2006. Aoste, Musumeci S.p.A., 361-370.
Rey P.-J., 2007. Le site Néolithique moyen du Dos de Borgaz (Aime) et la dynamique de l'occupation humaine en Tarentaise. In Besse M. dir. Sociétés néolithiques ; des faits archéologiques aux fonctionnements socio-économiques. Actes du $27^{\mathrm{e}}$ colloque Internéo, Neuchâtel 1-2 octobre 2005. Cahier d'Archéologie Romande, 108, 363-378.

REY P.-J., à paraître. Premières occupations de la montagne alpine sur les versants du Petit-Saint-Bernard. Bilan 2006 des sondages archéologiques sur le versant français. Etudes Savoisiennes. Université de Savoie.

Rey P.-J., Thirault E., 1999. Le peuplement des vallées alpines au néolithique : les exemples de la Maurienne et de la Tarentaise (Savoie). In Beeching A. dir. Circulations et Identités culturelles alpines à la fin de la Préhistoire. Programme CIRCALP 1997-1998. Travaux du CAP Valence, 2, 501-518.

Rey P.-J., TREFfort J.-M. avec coll. ANDRÉ I., à paraître. Il sito neolitico e protostorico del Châtelard de Bourg-SaintMaurice (Savoia) : insediamento a carattere difensivo e zona sepolcrale al piede del colle del Piccolo S. Bernardo. Atti del Convegno internazionale Il Piemonte e le Alpi occidentali all'alba della storia, Torino, Fondazione CRT, 13 e 14 aprile 2007.

Rey P.-J., Treffort J.-M., Moulin B., Oberlin C., Andre I., 2008. Archéologie des versants du Petit-Saint-Bernard; première approche de la dynamique de l'occupation humaine autour d'un grand passage alpin, de la Préhistoire au début du Moyen Âge. In Magny M., Desmet M. et Mocci F. dir. Du climat à l'homme; dynamique holocène de l'environnement dans le Jura et les Alpes. Actes de la table ronde du GDR JURALP, Aix-en-Provence novembre 2007. Collection Edytem, 6, 209-224.

Stuiver M., Reimer P.J., Bard E., Beck J.W., Burr G.S., Hughen K.A., Kromer B., Mac Cormac G., Van Der Plicht J., SPURK M., 1998. Intcal98 radiocarbon age calibration, 24000-0 cal BP. Radiocarbon 40, 1041-1083.

Thirault E., 2004a. Echanges néolithiques : les haches alpines. Montagnac : Monique Mergoil Ed. (collection Préhistoires, 10). $468 \mathrm{p}$.

Thirault E., 2004b. Le site néolithique de Bessans, Le Château (Savoie) et la question des armatures perçantes en roches polies dans les Alpes occidentales. In Dartevelle H. dir. Actes des $5^{\mathrm{e}}$ Rencontres Méridionales de Préhistoire Récente. Clermont-Ferrand, 8 et 9 novembre 2002. Cressensac : Préhistoire du Sud-Ouest, 421-444.

Thirault E., 2006. Bessans, La Teha (Savoie) : présence néolithique à haute altitude $(2250 \mathrm{~m})$ sur les itinéraires transalpins. Bull. SPF, 103(4), 797-799.

Venturino Gambari M., 2000. Forme e dinamiche degli insediamenti umani nel neolitico e nell'eneolitico. In Mercando L. et Venturino Gambari M. dir. Archeologia in Piemonte, vol. I La Prehistoria. Torino : Umberto Allemandi éd. 101-121.

Vital J., Bennamour P., Brochier J.-L., Chemin R., 2008. La grotte des Balmes à Sollières-Sardières. In Jospin J.-P. et Favrie T. dir. Premiers bergers des Alpes de la Préhistoire à l'Antiquité. Musée Dauphinois : Infolio. 90-91.

WinIGER A., 1994. Etude du mobilier néolithique de SaintLéonard «Sur le Grand Pré» (Valais, Suisse). Université de Genève DAE. Thèse de Doctorat. 3 vol., 511 p. 



\section{NEIGE et GLACE de MONTAGNE \\ Reconstitution, dynamique, pratiques}

\section{Sommaire}

Editorial

Introduction

\section{1 - Reconstitution}

Coutterand S. et al. - Le lobe glaciaire lyonnais au maximum würmien : glacier du Rhône ou/et glaciers savoyards ?

Ravanel L. et al. - Désenglacement du haut bassin versant du Vorz (massif de Belledonne, Isère), au Tardiglaciaire et à l'Holocène.

Rey P.-J. - Sociétés et fluctuations du climat dans les Alpes nord-occidentales au Néolithique moyen.

Le Roy et al. - Étude des fluctuations glaciaires du Petit Âge de Glace dans le Massif des Écrins : apports de la lichénometrie.

Kirkbride M.P. - Datation des moraines holocènes d'Islande par tephrochronologie : un état de l'art.

Le Roy et al. - La dendroglaciologie, ou l'apport de l'étude des cernes d'arbres pour la reconstitution des fluctuations glaciaires holocènes.

Rabatel A. - Évolution glaciaire dans les andes subtropicales chiliennes entre 1955 et 2007 : conséquences pour la ressource en eau.

Le Roy et al. - Un inventaire des aérophotographies du massif du Mont Blanc.

\section{2 - Dynamique}

Ravanel L. - Évolution géomorphologique de la haute montagne alpine dans le contexte actuel de réchauffement climatique.

Gruber S. - Le permafrost de haute montagne.

Deline P. et al. - L'Aiguille du Midi (massif du Mont Blanc) : un site remarquable pour l'étude du permafrost des parois d'altitude.

Saulnier G.-M. et al. - Un éléphant volant est-il un oiseau ? Perspectives pour l'observation hydrométéorologique des milieux de montagne.

Jobard S. - L'instrumentation du glacier du Baounet : quels apports pour la traçabilité des mesures environnementales ?

Moreau L. - L'exploration du cryokarst glaciaire et son intérêt scientifique pour l'étude du drainage des eaux de fonte.

Mazué R. et al. - Suivi de l'évolution de la couverture detritique d'un glacier noir par photo-comparaison : le glacier d'Estelette.

\section{3 - Pratiques}

Paccard P. - Réchauffement climatique et ressource neige en domaines skiables.

Gauchon C. - Les hivers sans neige et l'économie des sports d'hiver : un phénomène récurrent, une problématique toujours renouvelée.

Laslaz L. - L'exclusion des glaciers des zones centrales des Parcs nationaux de la Vanoise et des Écrins et leur équipement pour le ski d'été.

Cayla N. - Les sentiers d'interprétation glaciaire : des outils de valorisation différenciée des glaciers et de leur territoire.

Lambert R. - Cartozonage : de la carte au zonage du risque avalanche.

Moulin A. et al. - L'incertitude liée aux avalanches dans les Alpes du Nord : identifications et implications pour la gestion.

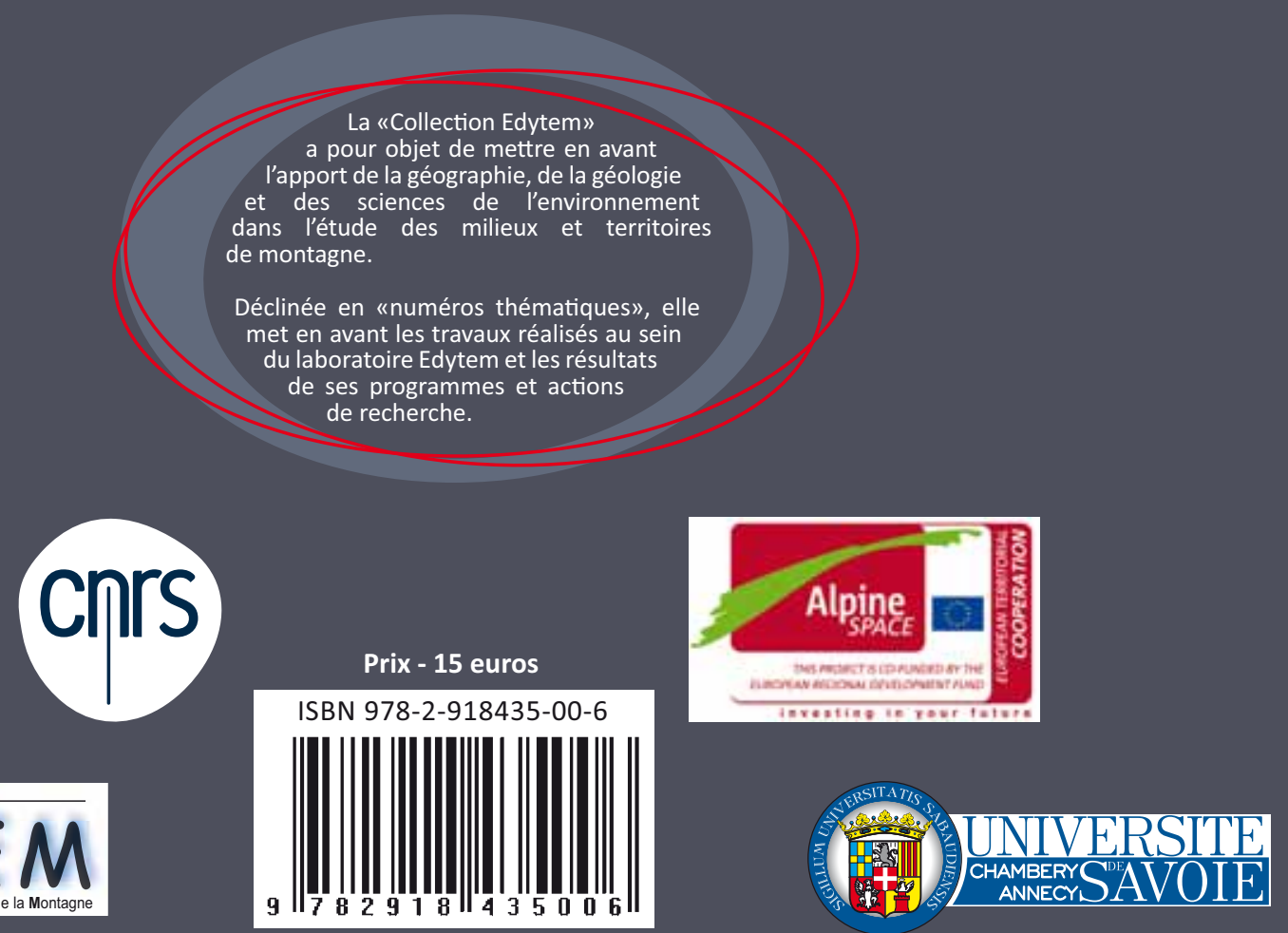

